\title{
ÜBER DIE ACHT SCHNITTPUNKTE DREIER OBERFLÄCHEN ZWEITER ORDNUNG.
}

\author{
Auszug eines Schreibens an Herrn H. G. Zeuthen \\ VON
}

H. SCHROETER

in BRESIAU.

...... Der schöne Satz, welchen Sie kürzlich im Anschluss an eine Arbeit des Herrn Dobriner in den Acta mathematica, Bd. 12, mitgetheilt haben und der gegenüber der Hesseschen Darstellung eine vollkommene Symmetrie liefert für eine Gruppe von acht associirten Punkten findet sich implicite ausgesprochen in einer Arbeit von A. Buchнеm: An extension of Pascal's theorem to space of three dimensions (Messenger, (2) Vol. 1 4, 74-75). Die Arbeit selbst habe ich nicht nachsehen können, weil sie auf der hiesigen Bibliothek nicht vorhanden ist; wohl aber findet sich in den 》Fortschritten der Mathematik», Bd. 16, S. 576, folgendes Referat:

»Wenn ein Achteck einer cubischen Raumkurve einbeschrieben ist, so sind die Schnitte der Gegenflächen die Erzeugenden eines Hyperboloids; d. h. also, nennt man die Ecken des Achtecks I 2345678 und bezeichnet durch 123 die Ebene durch die Punkte 1, 2, 3, so sind die vier Linien $(123,567)(23.4,678)(345,781)\left(45^{6}, 8\right.$ I 2$)$ die Erzeugenden eines Hyperboloids.) Glr. (Lp.)

Der Beweis, welchen ich mir vor mehreren Jahren von diesem Satze gemacht habe, setzt nichts weiter voraus, als dass durch die acht gegebenen Punkte sich Hyperboloide legen lassen, gilt also ebensowohl für 
acht Punkte einer Raumkurve dritter Ordnung, wie für eine Gruppe von acht associirten Punkten. Gestatten Sie mir, Ihnen denselben mitzutheilen:

Wenn acht Punkte des Raumes $1,2,3,4,5,6,7,8$ eine Gruppe von acht associirten Punkten bilden, also jede Oberfläche zweiter Ordnung, die durch sieben derselben geht, auch den achten enthalten muss, so wird ein Hyperboloid, welches die Geraden $|12||67|$ zu Erzeugenden hat und durch die Punkte 3, 4,5 geht, auch den Punkt 8 enthalten, also findet die Projectivität der beiden Ebenenbüschel statt:

$$
|\mathbf{1} 2|\left(345^{8}\right) \pi|67|\left(345^{8}\right) \text {. }
$$

Schneidet man die beiden projectiven Ebenenbüschel mit der Ebene [345], so erhält man zwei projective Strahlenbüschel, die einen Kegelschnitt erzeugen; auf diesem liegen die sechs Punkte:

$$
3,4,5, \quad(12,345)=\mathfrak{D}, \quad(67,345)=\mathfrak{D}_{1}, \quad(128,678,345)=\mathfrak{P}
$$

und bilden ein Pascal'sches Sechseck:

$$
345 D_{1} \mathfrak{B D}
$$

bei dem die drei Schnittpunkte der Gegenseiten

$$
\left(34, \mathfrak{D}_{1} \mathfrak{B}\right),(45, \mathfrak{Q}),\left(5 \mathfrak{D}_{1}, 3 \mathfrak{D}\right)
$$

auf einer Geraden $g$ liegen.

Diese drei Punkte der Geraden $g$ lassen sich nun anders ausdrücken, denn es ist offenbar

$$
\begin{aligned}
& \left|\mathfrak{O}_{1} \mathfrak{P}\right| \equiv|345,678|, \quad|\mathfrak{D} \mathbb{B}| \equiv \mid 345,8 \text { I } 2 \mid, \\
& \left|5 \mathcal{O}_{1}\right| \equiv|345,567|, \quad|3 \mathcal{O}| \equiv|123,345| \text {, } \\
& \left(34, \mathcal{D}_{1} \mathfrak{B}\right) \equiv(34,678), \quad(45, \mathfrak{O P}) \equiv(45,8 \text { I } 2) ;
\end{aligned}
$$

also liegen diese drei Punkte

auf der Geraden $g$.

$$
\begin{aligned}
& \mathfrak{\jmath}_{1}=(\text { I } 23,345,567), \\
& \mathfrak{z}_{2}=(34,678), \\
& \mathfrak{\jmath}_{4}=(45,8 \text { 1 } 2)
\end{aligned}
$$


Bezeichnen wir nun die vier Schnittlinien:

$$
\left\{\begin{array}{l}
l_{1}=|\mathrm{1} 23,567| \\
l_{2}=|234,678|, \\
l_{3}=|345,78 \mathrm{I}| \\
l_{4}=\mid 456,8 \text { I } 2 \mid
\end{array}\right.
$$

so ist ersichtlich, dass $g$ der Geraden $l_{1}$ begegnen muss, weil $\vec{\partial}_{1}$ in $g$ liegt und gleichzeitig in $l_{1}$; ferner begegnet $g$ der Geraden $l_{2}$, weil $z_{2}$ in beiden Geraden liegt; $g$ begegnet auch der Geraden $l_{4}$ weil $\delta_{4}$ in beiden Geraden liegt, und endlich begegnet $g$ auch der Geraden $l_{3}$, weil beide in derselben Ebene [345] liegen; also begegnet $g$ allen vier Geraden $l_{1} l_{2} l_{3} l_{4}$ gleichzeitig. Auf dieselbe Weise, wie wir die Gerade $g$ gefunden haben, können wir weitere Gerade derselben Eigenschaft finden, indem wir von einem andern durch die acht Punkte gelegten Hyperboloid oder von einer anderen aus demselben entspringenden Projectivität ausgehen. Dies geschieht am einfachsten dadurch, dass wir die Zahlen I, 2, 3, 4, 5, 6, 7, 8 cyclisch fortschreiten lassen, also als zweite Projectivităt wählen

$$
|23|(456 \mathrm{I}) \bar{\wedge}|78|(456 \mathrm{I}) \text {. }
$$

Dadurch erhalten wir eine neue Gerade $g^{\prime}$; die vier Geraden $l_{1} l_{2} l_{3} l_{4}$ gehen aber durch cyclisches Fortschreiten in einander über, bleiben also dieselben; wir können demgemäss acht Gerade $g$ angeben, welche gleichzeitig den vieren $l_{1} l_{2} l_{3} l_{4}$ begegnen, woraus denn die hyperboloidische Lage der vier Geraden $l_{1} l_{2} l_{3} l_{4}$ d. h. Ihr Satz folgt.

Breslau im September I 889 . 